\title{
A Comparison of Competitive and Cooperative Task Performance Using Spherical and Flat Displays
}

\author{
John Bolton, Kibum Kim and Roel Vertegaal \\ Human Media Lab \\ Queen's University \\ Kingston, Ontario, K7L 3N6 Canada \\ \{bolton, kibum, roel\}@cs.queensu.ca
}

\begin{abstract}
While large flat vertical displays may facilitate persistent public sharing of work, they may do so at a cost of limited personal display space when everyone can see each other's activity. By contrast, new form factors, such as spherical displays, support sharing display space by limiting the user's view to at most one hemisphere. In this paper, we investigate how different interactive large display form factors can support differences in sharing of information during competitive and cooperative task conditions. We implemented three different large display types: spherical, flat, and a flat display with divider. Results show that task performance of the flat display with divider did not differ significantly from that of the spherical display. Additionally, we implemented and compared three peeking techniques that facilitated sharing of information. Results show participants peeked significantly more in competitive tasks than they did in cooperative tasks. Usage of peeking techniques between the spherical display and the flat display with divider were similar, and distinct from that of the flat display. Not surprisingly, results show that the affordance of easily glancing at a partner's work on the flat display provided a significant advantage in cooperative tasks.
\end{abstract}

\section{Author Keywords}

Spherical Displays, Organic User Interfaces, Collaboration, Shared Space.

\section{ACM Classification Keywords}

H5.m. Information interfaces and presentation (e.g., HCI): Miscellaneous.

\section{General Terms}

Design, Human Factors, Experimentation.

\section{INTRODUCTION}

Large interactive displays allow multiple collocated users to collaborate, share information and work in parallel. Large

Permission to make digital or hard copies of all or part of this work for personal or classroom use is granted without fee provided that copies are not made or distributed for profit or commercial advantage and that copies bear this notice and the full citation on the first page. To copy otherwise, or republish, to post on servers or to redistribute to lists, requires prior specific permission and/or a fee.

CSCW 2012, February 11-15, 2012, Seattle, Washington

Copyright 2011 ACM 978-1-4503-1086-4/12/02...\$10.00.

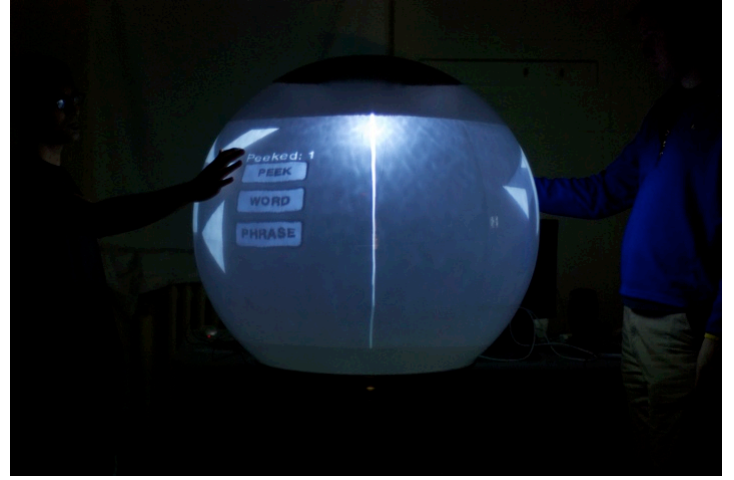

Figure 1. Spherical multi-touch display used in the experiment

flat displays typically do not prevent co-workers from viewing each partner's work, unless a physical barrier is introduced between them. In both collaborative and competitive settings, users often need to be able to keep certain information private $[3,13,16,18]$. The recent development of novel display form factors such as spherical displays [1, 2, 4, 12] offers new opportunities for designing sharing of display space, by presenting a natural visual barrier between users. While Benko [2] identifies spherical displays as a solution for single user scenarios that integrates both an interesting display form factor and input sensing, spherical displays also present a number of interesting properties that make them potentially suitable for cooperative as well as competitive tasks in multi-user conditions: (1) they increase the number of pixels on a display without compromising availability of personal physical space, by wrapping pixels around an optimal volume, (2) they offer more freedom in terms of users' physical body positions, with a smooth transition between shared and individual zones and (3) they enable curved data, such as satellite data images of the earth or panoramic visualizations to be mapped onto a volume without distortion.

In particular, spherical displays physically limit a user's view to at most one hemisphere, and naturally provide users with their own personal territories, without restricting the amount of data displayed (see Figure 1). This lets users maintain their own display space while still allowing for collaboration using a single communal display. Because of its geometry, users of a spherical display may have an 
intuitive understanding of what information might be shared, and what information might be private. For example, information at the edge of a user's view is easily shared. On the other hand, due to the visibility constraints of a curved surface, opposite work areas are naturally separated from each other, thus reducing the potential for interference.

Another interesting characteristic of a spherical display is that it presents pixels around a central point, providing no set or single location for a user's workspace. Depending on the task, users can walk freely around a spherical display to select the location or part of the data they wish to work on. For example, if users plan to work in a tightly coupled fashion, they can choose to stand next to each other in front of the sphere. By contrast, if users require privacy, they can arrange themselves to segment the display into two opposite hemispheres. Users can potentially move freely between these various modalities, something that may be difficult to do with traditional flat displays.

The continuous nature of a spherical display requires different approaches to presenting information. Because information viewable to a user of a spherical display is determined by the user's location, availability of information is largely determined by sharing physical territory. This contrasts with flat displays where, in general, all information is viewable to all users from all locations at all times.

Spherical displays wrap an extraordinarily large number of pixels into a space much smaller than that of an equivalent flat display. For example, a 3' diameter spherical display has a surface area equivalent to a 10' diagonal flat display. Not only does this facilitate collocated collaboration in small spaces, it also means the available pixels in a user's display space are within easy reaching distance, while at the same time those pixels remain largely invisible to other users.

Although individual interactions on spherical displays have been examined $[1,2,7,10,12,15]$, to the best of our knowledge, it has not been shown whether a spherical display's unique natural affordances for individual work spaces allow for a true advantage in a group activity, when compared to a flat vertical display. In this paper, we present an empirical evaluation of a low-cost multi-touch spherical display, compared with flat display form factors in cooperative and competitive tasks. To allow viewing restrictions similar to those observed in a spherical screen in our flat display condition, we introduced a third condition in which a physical divider split the view of the flat display between two users. Throughout competitive and cooperative tasks, we observed how users would peek to view invisible information on the other side of the display, using a set of widgets designed for this purpose. Our hypothesis was that in terms of performance and peeking technique usage patterns, the flat display with divider would be more similar in characteristics to the spherical display than to the flat display condition.

This paper is structured as follows: in the next section, we describe related work. We then discuss the details of our experiment, display apparatus and three peeking widgets. Finally, we discuss our findings and conclusions.

\section{RELATED WORK}

In this section we explore existing work on the development of various hemispherical, spherical and large public displays and interaction techniques on each display.

\section{Hemispherical Displays}

Companje et al. [7] developed Globe4D, an interactive four-dimensional globe. The system consisted of a hemispherical display that can be freely rotated along all axes. The earth application on this display allowed users to rotate a globe while shifting time, allowing them to see the movements of continental drift. Globe4D could be physically rotated, with the projected image kept in sync. However, it did not feature any other touch interaction techniques. This system is an early example of an interactive hemispherical display. It made use of its unique form factor to allow interaction with information that is not well suited for traditional flat displays.

\section{Spherical Displays}

Grossman et al. [10] developed a 3D geometric model building application to demonstrate multi-finger gestural interaction on a spherical volumetric display. The user's fingers were tracked using a Vicon motion tracking system. This camera-based motion tracking system requires an external setup. Interaction techniques were designed to make use of the unique features of volumetric displays, specifically, the 360-degree nature of the viewing volume. Their set of interaction techniques consisted of SurfaceBrowser for file management, model transformations, and techniques to combine models to create scenes. SurfaceBrowser could be rotated by scrubbing the non-dominant hand's index finger along the display surface, bringing hidden objects into view. They made the observation that the 360-degree visibility of information when using a volumetric display makes it useful for exploring collaborative multi-user interaction. Due to the unique properties of a 360-degree display, the authors mentioned the need to examine considerations of how users share space. Additionally, it was found useful for the SurfaceBrowser to show information on the inner surface of the display, in effect, reducing it from a 3D volumetric display to a $2 \mathrm{D}$ hemispherical display.

Holman \& Vertegaal [12] examined how a spherical display could be used to edit 3D NURBS (Non-uniform Rational Basis Spline) objects. Their display was projected upon using two external projectors. Multi-touch gestures were used to create curvilinear objects. Again, a Vicon motion capture system was used for tracking both the user's fingers and the display itself. This system was based on the metaphor of a pottery table, where the concept of spinning 
the display while using gestures such as poking or flattening allowed the manipulation of a 3D mesh. The nondominant hand was used for context, while the dominant hand was used for manipulation and deformation. A set of four interaction techniques was developed for use with the sphere. These techniques were spinning, poking, pulling, and mashing. They were used in combinations to produce specific effects. For example, a pull while spinning the display resulted in an extrusion along the entire circumference of the Blobject. This was an early example of the implementation of multi-touch gestures on a fully spherical display.

Benko et al. [1] developed a multi-touch spherical display that did not require external motion tracking, based on a 24" diameter Magic Planet system. To evaluate the system, they implemented a set of interaction techniques and reported on user behavior within four applications; a simple photo and video browser, an omni-directional data viewer, a painting application, and a Pong game. Interaction techniques consisted of dragging, local rotation, and scaling. Additional techniques such as object auto-rotation, flicking with inertia, and a command to send objects to the opposite side of the sphere were designed specifically for cooperative purposes. During their observations, they found that data spanning the entire display was problematic with multiple users. Individual users could not see others touching the display and users often became confused when seeing the results of other users' interactions. As a consequence, users would compete for control of the display. They concluded that limiting the consequences of a user's action to a specific area might be necessary. Additionally, they found that users had no master position around the sphere, and instead picked a location at random. However, they found that a single user would not move extensively from their initial position during use. Their observations seem to indicate that a spherical display presenting, at most, one hemisphere to a user is an important design consideration. Users still chose a specific work area when using a spherical display. This indicates that implementing methods for viewing information on the non-visible hemisphere is an important consideration for applications on spherical displays. We implemented peeking interfaces on our system to allow users to view the information on the non-visible hemisphere.

\section{Large Public Displays}

Vogel \& Balakrishnan [19] demonstrated an interactive large display supporting public and personal information, while allowing users to move seamlessly from implicit to explicit interactions. A framework of interaction phases was developed, consisting of ambient display, implicit interaction, subtle interaction, and personal interaction. During the personal interaction phase, direct touch was used to manipulate information items and provide additional detail. In this phase, the user's body helped occlude their personal information. Additionally, privacy gestures could be used to hide notifications and display elements, preventing the display from entering the personal interaction phase. Their observations indicated that privacy is an important consideration when designing interactive public large flat displays. They suggested that occlusion provided by a user's body was not sufficient when displaying sensitive information and that privacy design should be a major focus of production level displays.

Tsandilas \& Balakrishnan [17] examined techniques for reducing spatial interference between users on shared displays. Four methods were considered, including object ownership, and split displays. With a shared screen, users had access to the entire display, but could only manipulate objects they owned. This allowed users to move freely around the display and control their own boundaries. However, this approach is dependent on the social situation. In some cases, a single user may try and control a disproportionately large area of the screen. Additionally, this does not take into account a user's desire for privacy. By contrast, a split display removed interaction conflicts between two users but limited users to an explicitly defined area. Spherical displays naturally provide a split screen method of interaction due to their visibility constraints. As a user's position is generally fixed around a spherical display, there are few disadvantages to restricting a user to an explicitly defined area.

\section{EXPERIMENT DESIGN}

Many previous studies in Computer Supported Cooperative Work (CSCW) show that when users can see where others are working, it becomes easier to establish common ground in cooperative tasks $[8,9,14]$. However, there are also complicated situations, particularly in competitive gaming, where participants may not wish to share all their information with each other but still need to check their partner's progress for strategic purposes. It is important to understand this fundamental tradeoff between sharing information, actively or passively, and preserving privacy as being juxtaposed. To better understand the effect of display form factors on this information sharing tradeoff in collocated tasks, we designed an experiment in which we investigated group activities in three single display groupware settings; a large spherical display, a large flat vertical display, and a large flat vertical display with divider.

As Benko et al. [1] pointed out, there are some characteristics of spherical displays that may make them suitable for shared tasks. Collaborators can, for example, stand opposite to each other, providing the potential for sharing of eye contact and facial expressions, if the sphere is small and low. To provide a leveled playing field, and to allow for comparative screen real estate with the large flat display conditions, we constructed a large spherical screen that allowed collaborators to only sense each partner's presence, but not to see each other's face. We also introduced a physical divider that split the flat display and used this as a baseline of our experiment, allowing for 
similar visibility, and thus privacy settings, as the spherical display.

In order to help participants access their partner's screen, which was not readily visible on the spherical and the flat with divider displays, we designed a number of peeking techniques. Those techniques allowed participants to easily browse their partner's workspace if necessary. Participants used three peeking techniques (i.e., button, windowing and scroll) as well as conversing with their partner or physically walking around the display to access their partner's work. Frequency of peeking techniques allowed us to measure the effects of visibility characteristics of different display conditions on group tasks.

Tradeoffs between preserving privacy and sharing a visual workspace may vary with respect to the privacy characteristics of the task. We therefore developed two different group scenarios: one cooperative and one competitive task setting. In the cooperative tasks, participants solved a puzzle together as a team. In competitive tasks, participants competed with their partners but also worked with them-the task was designed as a contest that still required the sharing of information. This cooperative and competitive task design enabled us to create collaboration styles where participants were required to maintain a balance of preserving their own work and sharing their partner's work actively or passively.

There has been some suggestion that spherical screens may be inherently more suited to $3 \mathrm{D}$ tasks, giving them an inherent advantage over flat panel displays [10]. To not positively bias the spherical condition, we decided to design our task using more common 2D graphics. Tasks included a mix of graphical object manipulation and text retrieval tasks common to most Graphical User Interfaces (GUIs). A second reason for using a graphical game was that it made it more difficult to verbally share information about the figures, thus encouraging participants to peek at each other's display instead.

We used a $3 \times 4 \times 2$ within-subjects factorial design, with three factors: display type (3 levels consisting of spherical, flat, and flat with divider), peeking technique (4 levels consisting of button, windowing, scroll, and physical peeking), and collaboration style (2 levels consisting of cooperative and competitive). In the flat display condition, physical peeking required participants to simply turn their head. In the spherical and flat display with divider conditions, the participants walked around the display to view their partner's work. Each group of two participants completed the 24 tasks in a single two-hour session. To minimize any practice or carry-over effects, the order of the conditions was counterbalanced using a Latin square.

\section{EXPERIMENT METHODOLOGY}

We conducted a controlled laboratory experiment to compare the effects of display curvature and peeking techniques on cooperative and competitive collaboration

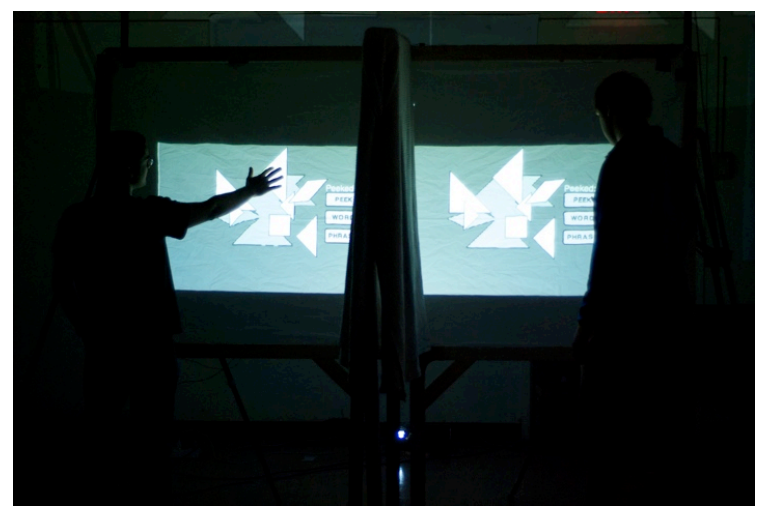

Figure 2. Large flat vertical display with divider used in the experiment.

styles. This section describes the apparatus and methodologies used to conduct our experiment.

\section{Display Apparatus}

The experiment was run on three different prototype displays; a spherical display, a large flat vertical display, and a large flat vertical display with divider.

\section{Spherical Display}

Our spherical display surface consisted of a 36 " diameter hollow sphere made of acrylic, with a 19" hole cut in the bottom (see Figure 1). The acrylic was sandblasted to create a diffuser on the inside, allowing it to act as a projection surface. This also allowed it to act as a diffuse illumination surface for infrared light, which allowed multi-touch detection. The projector was mounted at the bottom of a base, an oil drum commonly known as a burning barrel, using a projector mount inside of the barrel. The projector was aimed upwards in order to project off an 18 " hemispherical mirror mounted inside the top part of the sphere. This mirror projected light to the rest of the surface of the display. A custom Quartz Composer patch provided spherical distortion to the graphics so as to ensure graphics appeared undistorted after projection onto the sphere's surface. Infrared illuminators were pointed at the inside of the acrylic sphere and arranged to spread light evenly across the surface. Two SONY EyeToy cameras with wideangle lenses were modified as infrared cameras. These were placed inside the sphere pointing towards each hemisphere. We used a diffuse illumination approach to register multitouch points by the fingers on the surface of the sphere.

\section{Large Flat Vertical Display}

The large flat vertical display consisted of an 8' by 4' piece of $1 / 4$ " plexiglass mounted inside a wooden frame. Six $25 \mathrm{~mW}$ infrared lasers with line generating lenses were mounted around this large piece of plexiglass, creating a plane of infrared light that was slightly elevated off the surface of the plexiglass. A large rear-projection screen was fastened to the back of the plexiglass to provide a diffuser. A SONY EyeToy camera with a custom lens that had been modified to allow it to sense infrared light was placed behind the plexiglass for touch detection. 


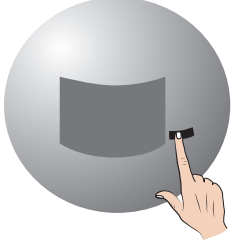

(a) Peek Button

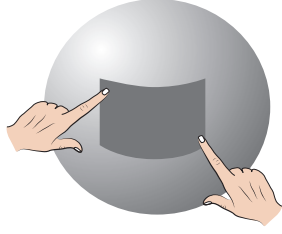

(b) Windowing

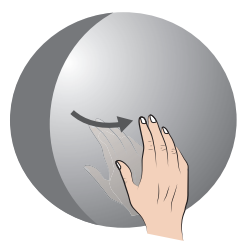

(c) Scroll
Figure 3. Peeking techniques used in the experiment.

For the flat large vertical display with divider setting, we mounted a curtain as a divider in the middle of the large flat vertical display (see Figure 2). This blind was used to prevent participants from being able to observe each other's workspaces as well as each other's bodies, in one condition of the experiment.

\section{Touch Detection on the Display}

Touch detection was accomplished using the open source project Community Core Vision. An unmodified version was used for the flat screen hardware condition while a multi-cam version was used for the sphere. Care was taken that the final resolution of touches was identical in all display conditions.

\section{Peeking Techniques}

Participants were instructed that they would converse and physically walk around the display to see their partner's work but could also use peeking techniques as much as they pleased, in all display settings. We designed the following three peeking techniques to facilitate convenient access to a partner's workspace (Figure 3):

\section{Peek Button.}

A large button, labeled "PEEK", was placed to the right of the work area. When touched, this button opened a window showing the partner's workspace. The window was of fixed size, and covered the entire workspace. The window was removed by touching the "PEEK" button a second time.

\section{Windowing}

Here, participants used a bimanual pinch gesture to open a variable-size window into the partner's work area. Touches were used to form the top left and the bottom right corner of this window, allowing the participants to peek only at a portion of the partner's workspace.

\section{Scroll}

A scroll window was triggered when participants placed their hand flat onto the display surface. This scrolling window was of fixed vertical size, but brought more of the partner's workspace into view as the participants moved their hand horizontally away from left to right or to the opposite direction. This potentially allowed participants to view the entire workspace of the partner.

In all cases, changes were only visible to the participant performing the gesture, but the partner was informed of the peeking act by increasing a "Peeked" counter to the side of his workspace (see Figures 1 and 2). We expected that there would be a different preference for the chosen peeking

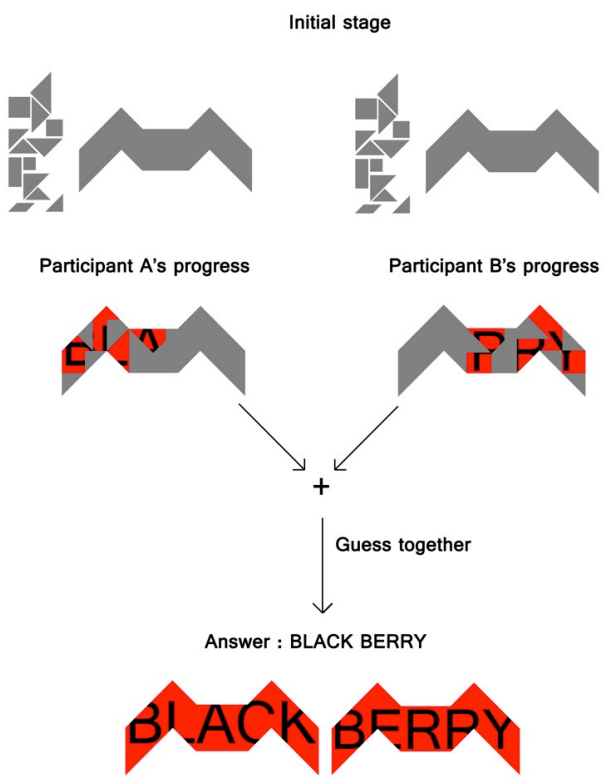

Figure 4. Tangram phrase guessing game (in cooperative condition).

techniques on each display and the frequencies of usage for each peeking technique might vary depending on the display form factor.

\section{Task}

For our experimental task, we chose an electronic variant of the Tangram game, which is widely used to explore the creation and maintenance of common ground in CSCW and psycholinguistic literature $[5,16,20]$. The original Tangram game requires users to arrange a number of different geometric pieces into various shapes within a silhouette of the target shape provided. We modified this basic Tangram game by combining it with a phrase-guessing game in order to increase the need for peeking and communication between participants (Figure 4).

The goal of our modified Tangram game was to find a hidden two-word phrase inside the solution silhouette. When a participant moved a piece to the correct position on the Tangram silhouette, this piece revealed a portion of a word. Each participant received a different word, which together formed a phrase. Our Tangram phrase guessing game required participants to work individually as well as collaboratively to achieve the task goal. Participants would not need to assemble all pieces of the Tangram puzzle if they collaborated and guessed the phrase based on a partially solved puzzle. Both participants started with the same puzzle, displayed in different hemispheres on the spherical display or in a separate working area on the flat display. Participants were co-located in all conditions. They were able to use both hands to move pieces around their workspace. To ensure that any geometric distortion of the 2D graphical elements on a spherical screen would not negatively impact performance, all pieces were in the correct orientation, and did not require rotating the shape. When a piece was within 10 pixels of the correct location it would snap into place upon release of the piece. 
During the game, participants were allowed to talk to each other as well as walk around to their partner's workspace to view his or her Tangram. In addition, they were allowed to use one of the three peeking techniques, based on the experiment conditions, to view what was on their partner's screen. This allowed us to investigate whether the peeking techniques were efficient and effective in use.

In the cooperative task, participants completed the Tangram game together as a team. They could share their partner's progress and help the partner move pieces or guess the solution. There were two components to completing a game: finding a word and finding a phrase. After a participant found a word, he or she was asked to click a button labeled "WORD". The round was complete when both participants had hit the "PHRASE" button and provided the correct solution (see Figures 1 and 2). This setup allowed us to measure both individual and combined task performance.

In the competitive task, the goal was to be the first to correctly guess the two-word phrase. Participants might monitor their partner's progress and take advantage of peeking to get a hint of how to assemble the pieces or in order to guess the phrase first. As with the cooperative condition, after a participant found a word, he or she was asked to click a button labeled "WORD". The winner was decided when one of the participants hit the "PHRASE" button and provided the correct solution by writing it on a note. The other participant continued work on the task until $\mathrm{s} /$ he was able to guess the phrase.

\section{Participants}

Fourteen groups of participants (mostly university students, 12 males and 16 females), all paid volunteers, worked in pairs to complete Tangrams in the three display conditions. Some partners knew each other and had previously worked together. The average age of the participants was 21.8 years, with a range between 18 and 36 years old. All participants reported some familiarity with multi-touch UIs.

\section{Procedure}

Before beginning the experiment, participants were asked to fill out a brief demographic survey as well as a consent form. They were then given written instructions for each task on a piece of paper.

Following this, participants underwent a training period for each display system. This allowed them to get a feel for the game and the different methods of viewing each partner's information. During the training session, participants were required to practice each peeking technique at least five times and were allowed to ask for any clarification. The training session took a total of 12 minutes of instruction on the game procedures in both cooperative and competitive conditions. Of this time, about 7 minutes was used to practice the different peeking techniques.

After the training session, participants were asked to complete the Tangram games on three different touch

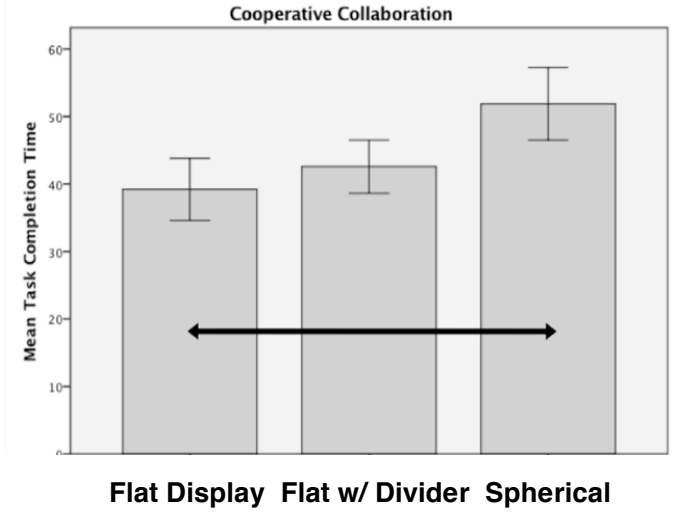

Figure 5. Task completion times in the cooperative tasks (arrow indicates significance between flat and spherical display).

screens: a spherical display, a large flat vertical display, and a large flat vertical display with divider. They proceeded to play 12 games in the cooperative condition and 12 games in the competitive condition. Participants were given a maximum of two minutes to guess the phrase. All conditions were presented in a counterbalanced order based on Latin squares.

To motivate efficient and accurate performance, the participants of the fastest team from all groups were rewarded with a $\$ 20$ gift card for the cooperative condition. Additionally, the fastest individual participant of all groups in the competitive condition was rewarded with a $\$ 10$ gift card.

After each display condition (i.e., spherical, flat, and flat with divider), participants were asked to finish the NASA task load index (NASA-TLX), which is a subjective, multidimensional assessment tool designed to obtain workload estimates from users. Finally, when participants completed all conditions, an experimenter conducted an interview with participants in order to collect general comments on their experience during the experiment. All experimental sessions were recorded on video to allow transcription of discussions between participants.

\section{RESULTS}

Results were calculated over 13 groups. Data from one group was discarded because they did not follow instructions and played the game cooperatively during the competitive condition. All results were analyzed using a within-subjects repeated measures analysis of variance (ANOVA), evaluated at an alpha level of .05.

\section{Task Completion Time}

Task completion time was defined as the slower of the two participants' time to the final phrase in the cooperative collaboration condition and as the faster of the two participants' time to the final phrase in the competitive collaboration condition. Our first analysis, which was a three-way ANOVA across collaboration styles, display types, and peeking techniques, showed a significant main effect of collaboration styles, but no significant effect for display types or peeking techniques: collaboration styles, 


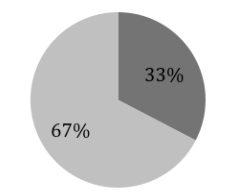

- Cooperative Collaboration

- Competitive Collaboration

(a) by collaboration style

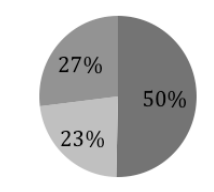

- Flat Display

- Spherical Display

(b) by display type
- Flat Display w/ Divider

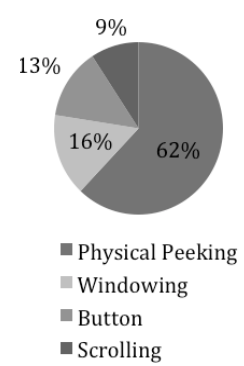

(c) by peeking technique
Figure. 6 Frequency of peeking.

$F(1,6)=18.62, p<.01$; display types, $F(2,12)=3.17, \mathrm{p}=.08$; and peeking techniques, $F(3,18)=1.55, \mathrm{p}=.24$. Participants completed the task faster in a cooperative collaboration style than they did using a competitive collaboration style.

For our next analysis, we separated out the collaboration styles to see if they were affected differently by the form factor of the display. A two-way ANOVA showed a significant main effect of display types on performance in cooperative collaboration $(F(2,13)=3.74, p<.05)$ but not in competitive collaboration $(F(2,16)=2.99, \mathrm{p}=.08)$. Post-hoc pairwise Bonferroni corrected comparisons of cooperative collaboration showed this difference to lie between the flat display and the spherical display $(p<.04)$. It also showed there were no significant differences between the flat display with divider and the spherical display (see Fig. 5).

\section{Peeking}

To examine the effects of visibility of information, we analyzed how often the participants peeked during the experiments. Figure 6 shows the frequency of each peeking technique broken down by experiment factors. Results were analyzed by a within-subjects repeated measures ANOVA across collaboration styles, display types, and peeking techniques. There were significant main effects for all three factors (i.e., collaboration styles, display types and peeking techniques). Participants peeked more than twice as much in the competitive collaboration condition as they did in the cooperative collaboration condition $(\mathrm{F}(1,6)=34.51, p<.001)$. Participants also peeked almost twice as much on the flat display as they did on the spherical display or on the flat display with divider $(\mathrm{F}(2,26)=8.52, p<.002)$. Of the four different peeking techniques, physical peeking was preferred $(\mathrm{F}(3,39)=85.58, \quad p<.001)$. It seems that the frequency of peeking was inversely proportional to the physical effort required-Turning the head in the flat display condition required less effort than traveling around the display in the spherical display or the flat display with divider conditions, resulting in more peeking using the flat display. Also, competitive conditions increased peeking and appeared to reduce conversation between the partners as compared to cooperative conditions.

Figure 7 shows the frequency of use of each peeking technique as a percentage of the total number of peeks per

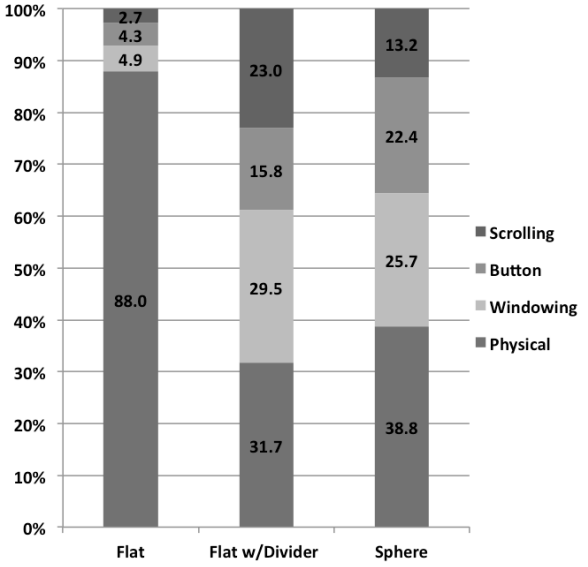

Figure 7. Peeking techniques preferences.

display type. There was a clear preference for physical peeking with the flat display. However, interestingly, participants used the software-based peeking techniques too on the flat display although they could easily see the partner's workspace by turning their head. This might be due to the novelty of these software-based peeking techniques, or because they wanted to peek without attracting any partner's attention. There was a slight preference for the windowing technique on the spherical display $(26 \%)$ and on the flat display with divider (30\%). The preference for the windowing peeking technique may indicate that participants tried to minimize the amount of their own workspace that was obscured.

\section{Physical Peeking}

In addition, we isolated the case where participants were only allowed to physically peek. This means that none of the software peeking techniques (i.e., button, windowing, scrolling) were provided but participants could physically move around or turn their head to see their partner's workspace. This separate analysis of physical peeking helped us to understand participants' natural interaction with their partner in each display condition. Additionally, it allowed us to examine, in general, whether providing different peeking techniques changed participants' behavior in cooperative and competitive collaborations.

A two-way ANOVA showed significant main effects for display types and collaboration styles on the frequency of physical peeking $(F(2,6)=10.86, p=.010$ for displays, $F(1,3)=24.4, p=.016$ for styles) and the task completion time $(F(2,22)=6.36, p=.007$ for displays, $F(1,11)=8.04$, $p=.016$ for styles). Participants peeked less and completed tasks significantly faster in cooperative conditions (average 5.4 peeking counts, 44.2 seconds in task completion time, $p=.016)$ than in competitive conditions (8.7 counts, 54.9 seconds).

A Bonferroni post-hoc test revealed that the time to complete the task was significantly faster on the flat display (42.1 seconds, $p=.008$ ) compared to the spherical display 


\begin{tabular}{lcccc}
\hline & $\begin{array}{c}\text { Spherical } \\
\text { Display }\end{array}$ & $\begin{array}{c}\text { Flat } \\
\text { Display }\end{array}$ & $\begin{array}{c}\text { Flat w/ } \\
\text { Divider }\end{array}$ & \\
\hline $\begin{array}{c}\text { How physically } \\
\text { demanding was } \\
\text { the task? }\end{array}$ & $\begin{array}{c}6.50 \\
(1.07)\end{array}$ & $\begin{array}{c}4.42 \\
(0.82)\end{array}$ & $\begin{array}{c}5.73 \\
(0.96)\end{array}$ & $p=0.05$ \\
\hline $\begin{array}{c}\text { How hurried or } \\
\text { rushed was the } \\
\text { pace of the task? }\end{array}$ & $\begin{array}{c}11.69 \\
(1.19)\end{array}$ & $\begin{array}{c}9.27 \\
(1.39)\end{array}$ & $\begin{array}{c}9.58 \\
(1.33)\end{array}$ & $p=0.02$ \\
$\begin{array}{c}\text { How hard did } \\
\text { you have to work } \\
\text { to accomplish } \\
\text { your level of } \\
\text { performance? }\end{array}$ & $\begin{array}{c}10.19 \\
(0.79)\end{array}$ & $\begin{array}{c}7.65 \\
(1.01)\end{array}$ & $\begin{array}{c}8.31 \\
(0.99)\end{array}$ & $p=0.01$ \\
\hline
\end{tabular}

Table 1. Mean ratings (s.e.) for physical demand, temporal demand, and effort for each display condition. Lower scores are better.

(55.3 seconds). There were no statistically significant differences for task completion time between the flat display with divider (51.2 seconds) and the flat display $(\mathrm{p}=.23)$ or between the flat display with divider and the spherical display $(\mathrm{p}=.64)$. For the frequency of peeking, no statistical difference was found in pairwise comparisons of displays.

Overall, the result of physical peeking showed similar patterns to the cases of the software-based peeking techniques: (1) participants peeked less and completed tasks faster in cooperative collaboration than in competitive collaboration, and (2) peeked more on the flat display (14.1 times) than on the spherical display (3.9 times) or on the flat display with divider (3.1 times).

\section{Subjective Preferences}

In the post-experiment questionnaire, participants were asked to select which display they preferred. Six scales of the NASA TLX, mental demand, physical demand, temporal demand, performance, effort, and frustration were measured on a scale from $0-20$ where 0 was a low and 20 was a high rating for that factor.

Table 1 shows the data summary for the mean of physical demand, temporal demand, and effort on each display. A Friedman test indicated that there were statistically significant differences in physical demand $\left(\chi^{2}(2)=6.000\right.$, $p=0.050)$, temporal demand $\left(\chi^{2}(2)=7.537, p=0.023\right)$ and effort $\left(\chi^{2}(2)=8.521, p=0.014\right)$ depending on the type of display.

Post-hoc analysis with Wilcoxon Signed-Rank Test was conducted with Bonferroni correction applied, resulting in the significance level set at $\mathrm{p}=0.017$. For physical demand, there were no significant differences between the flat display with divider, and the spherical display or the flat display. However, there was a statistically significant reduction in physical demand in the flat display vs. the spherical display $(\mathrm{Z}=-2.551, p=0.011)$. For temporal demand, there were statistically significant reductions on the flat display $(\mathrm{Z}=-2.378, p=0.017)$ and the flat display with divider $(Z=-2.641, p=0.008)$ versus the spherical display. However, there was no significant difference in temporal demand between the flat display and the flat display with divider. For effort, there were no significant differences between the flat display with divider, and the spherical display or the flat display. However, there was a statistically significant reduction in effort in the flat display vs. the spherical display $(\mathrm{Z}=-2.838, p=0.005)$.

\section{OBSERVATIONS}

We also observed differences in how participants interacted during each condition. In particular, we analyzed transcripts from our interviews and video footage, focusing on how participants collaborated.

\section{Collaboration on the Flat Display}

P1: "When we were trying to solve our puzzles together it was a lot easier when there was no screen in the way."

The ease with which participants viewed each partner's information on the flat display enabled both participants to be more aware of each other's context than on the spherical display or on the flat display with divider. Participants often conversed based on updates of their partner's work. This real-time monitoring of awareness information was not as straightforward with the non-flat display types.

\section{Collaboration on the Spherical Display or on the Flat Display with Divider}

P11: "Okay the two triangles are on the left side, the two big ones. The square is almost at the end of the G shape. My word is 'Roots'."

Similar to the spherical display, when using the flat display with divider, the context for participant's advice was not inherently visible. As a result, participants gave detailed instructions. In contrast to deictic references such as "this" or "that", which were more frequently observed in the flat display condition, participants described specific pieces, ensuring that the other partner could clearly understand their advice without having access to a view of the puzzle. In the above case, participant 11 was providing the correct location for two pieces. He made clear which pieces he was referring to by describing them and explaining where to move them. Following this, he provided his WORD to help the partner guess her WORD correctly and the PHRASE.

\section{Verbal Communication}

P12: "I found I was much more vocal, I suppose much more for communicating rather than just looking at his screen. We had a conversation while we worked collaboratively."

Although not always used by all groups, verbal communication was the predominant method of sharing information. Certain groups maintained a conversation while collaborating, constantly updating each other on the visible portions of their WORD, the correct position for various pieces, and guesses of what their WORD or the PHRASE might be. Some groups observed sharing 
strategies, which providing suggestions for which pieces to move into position upon starting the puzzle.

Peeking on Competitive and Cooperative Collaborations P11: "When I'm collaborating I can just ask her but when I'm competing I can't ask her so I have to do it sneakily (i.e., peeking)."

During the competitive collaboration, participants were much more likely to use peeking techniques. As emphasized above, participants still wanted to view partner's information but were not willing to share information with their partner. The peeking techniques became a method of sneaking information during the competitive collaboration, in contrast to the sharing that was common during the cooperative collaboration.

P4: "I can see what she's doing and she can see what I'm doing and we don't necessarily have to communicate verbally."

Although verbal communication was a common method of sharing information between participants when collaborating, sometimes it was not necessary. By providing the partner with access to all information using peeking techniques, participants didn't feel a need to constantly update the partner verbally on progress, as a quick glance was sufficient for sharing the partner's work.

\section{DISCUSSION}

For the cooperative task condition, the flat display performed better than a spherical display or the flat display with divider. Participants completed cooperative tasks on the spherical display $32 \%$ slower than they did on the flat display. However, performance of the spherical display was not significantly different from that of the flat display with divider. As expected, easy sharing of information on the flat display was beneficial in the cooperative condition and, for this reason, participants could complete the tasks faster using the flat display than using the spherical display or the flat display with divider. As for the competitive task condition, contrary to expectations, display form factor had no significant effect on performance.

Although participants were provided with various peeking techniques to access the information on the partner's screen, we observed that participants were more likely to view their partner's information by turning their head or walking around. They used this physical peeking significantly more than all other methods of peeking. In the flat display condition, physical peeking tended to consist of head rotation, whereas in the other display conditions, physical peeking required the participant to walk around to the partner's side of the display to view what was on his/her screen, impacting performance. In the spherical display condition, participants took longer to walk around than in the flat display with divider condition.

Despite the fact that a participant could not see all the details on the partner's screen on the spherical display or on the flat display with divider, they could discuss what they were doing and where they were working, if necessary. This method of discussion seemed to be faster and relatively easier than using the software-based peeking techniques to view the other screen. Verbal conversations were the prevalent form of communication in all three display conditions. According to Clark's principles of least collaborative effort in language use, people apparently do not like to expend more effort than they need to get their addressees to understand them [6]. Such a principle helps account for the phenomenon of the prevalence of verbal communication offset use of the software-based peeking techniques in all three different display conditions.

In single display groupware, partitioning of workspace content and reduction of dominant behavior by one participant has often been achieved by establishing separated personal territories [16]. However, we found that the natural division of workspace on the spherical display and the flat display with divider did not eliminate dominant behavior. We observed several cases in which a participant who had completed his part of the puzzle moved to the partner's screen and took control of her Tangram pieces. This behavior was contrary to our expectations for the spherical display or the flat display with divider's ability to maintain privacy but it suggested an alternative method of tight collaboration.

When using the flat display, maintaining an awareness of the other participant was relatively easy, natural, spontaneous, and unforced [11]. Unfortunately, this awareness was much harder to maintain in the spherical display and the flat display with divider conditions, and as a result, it was often difficult to determine what the other participant was doing. Our peeking techniques on the spherical display and on the flat display with divider involved a kind of workspace awareness that was forced and punctuated rather than natural and smooth. The participants had less awareness about the other person's activities in those display conditions than they did with the flat display. There was no difference in performance between the spherical display and the flat display with divider, but significant differences with the flat display. It showed that collaboration on large displays was primarily affected by the affordance of awareness, and not the display shape itself.

In terms of task performance, we received mixed results: As we expected, participants could finish the task faster when they cooperated than when they competed, but surprisingly the display type did not significantly affect task performance. However, when analyzing the effects of the displays in detail, by separating cooperative and competitive tasks, we found that the flat display was superior to the spherical display in the cooperative task. This result reinforces the validity of previous $\mathrm{CSCW}$ studies in the effect of visual sharing in cooperative tasks $[8,9,14]$. On the other hand, we were not able to find that the spherical display's natural affordances for individual workspaces allowed for a true advantage in the competitive 
tasks, when compared to the flat display or the flat display with divider.

One limitation of this study is that we used 2D graphical data on a spherical screen and the task was confined to one factual task rather than a richer array of more complex collaboration in a real-world environment. If the task had required interacting with 3D graphical data and frequent translation between public and personal space, we might have obtained different results. Our prototype and initial user experiences were encouraging and a next step is to visualize and interact with $3 \mathrm{D}$ models within the display. We are currently working on interaction techniques for 3D objects on spherical and cylindrical volumetric displays [4].

\section{CONCLUSIONS}

In this paper, we investigated how different interactive large display form factors might naturally support differences in sharing of information during competitive and cooperative task conditions. We compared performance in a two-person Tangram game between three display conditions: a spherical, a flat display, as well as a flat display with a physical divider between participants. To explore different degrees of sharing, we implemented three peeking techniques that facilitated sharing information: a button, a windowing, and a scrolling technique. The flat display condition performed better than the spherical display condition in a cooperative task setting. However, performance of the spherical display was not significantly different from that of the flat display with divider. In competitive tasks, display type had no significant effect. On the other hand, display type affected both the quantity and mix of peeking techniques used. Usage of peeking techniques between the spherical display and the flat display with divider were similar, and distinct from that of the flat display. Participants peeked significantly more in competitive tasks than they did in cooperative task. Our experiment result suggested that the affordance for the awareness of a partner's workspace on the flat display might have a greater significant advantage in cooperative tasks, compared to the spherical display's natural affordance for private workspaces.

\section{REFERENCES}

1. Benko, H., Wilson, A. D., and Balakrishnan, R. Sphere: multi-touch interactions on a spherical display. In Proc. UIST, (2008), 77-86.

2. Benko, H. Beyond flat surface computing: challenges of depth-aware and curved interfaces. In Proc. MM, (2009), 935-944.

3. Birnholtz, J. P., Grossman, T., Mak, C., and Balakrishnan, R. An exploratory study of input configuration and group process in a negotiation task using a large display. In Proc. CHI, (2007), 91-100.

4. Bolton, J., Kim, K. and Vertegaal, R., SnowGlobe: A Spherical Fish-Tank VR Display. In Proc. CHI (2011), 1159-1164.
5. Clark, H. Using Language. Cambridge University Press, Cambridge, MA, USA, 1996.

6. Clark, H. and Brennan, S. Grounding in communication. In L.B. Resnick, J.M. Levine, \& S.D. Teasley (Eds.). Perspectives on socially shared cognition. Washington: APA Books. 1991.

7. Companje, R., van Dijk, N., Hogenbirk, H., and Mast, D. Globe4D: time-traveling with an interactive fourdimensional globe. In Proc. SIGGRAPH. (2007), 26.

8. Fussell, S.R., Kraut, R.E., and Siegel, J. Coordination of Communication: Effects of Shared Visual Context on Collaborative Work. In Proc. CSCW, (2000), 21-30.

9. Gergle, D., Kraut, R. E., and Fussell, S. R. Action as language in a shared visual space. In Proc. CSCW, (2004), 487-496.

10. Grossman, T., Wigdor, D., and Balakrishnan, R. Multifinger gestural interaction with $3 \mathrm{~d}$ volumetric displays. In Proc. UIST, (2004), 61-70.

11. Gutwin, C. and Greenberg, S. A Descriptive Framework of Workspace Awareness for Real-Time Groupware. Journal of Computer Supported Cooperative Work 11, 3 (2002), 411-446.

12. Holman, D. and Vertegaal, R. Organic user interfaces: designing computers in any way, shape, or form. Communication of ACM 51, 6 (2008), 48-55.

13. Hudson, S. E. and Smith, I. Techniques for addressing fundamental privacy and disruption tradeoffs in awareness support systems. In Proc. CSCW, (1996), 248-257.

14. Kraut, R., Fussell, S., and Siegel, J. Visual Information as a conversational resource in collaborative physical tasks. Human-Computer Interaction 18, 1 (2003), 13-49.

15.Lin, J., Chen, Y., Ko, J., Kao, H., Chen, W., Tsai, T., Hsu, S., and Hung, Y. i-m-Tube: an interactive multiresolution tubular display. In Proc. MM, (2009), 253260.

16. Scott, S. D., Sheelagh, M., Carpendale, T., and Inkpen, K. M. Territoriality in collaborative tabletop workspaces. In Proc. CSCW, (2004), 294-303.

17. Tsandilas, T. and Balakrishnan, R. An Evaluation of Techniques for Reducing Spatial Interference in Single Display Groupware. In Proc. ECSCW, (2005), 225-245

18. Tse, E., Histon, J., Scott, S. D., and Greenberg, S. Avoiding interference: how people use spatial separation and partitioning in SDG workspaces. In Proc. CSCW, (2004), 252-261.

19. Vogel, D. and Balakrishnan, R. Interactive public ambient displays: transitioning from implicit to explicit, public to personal, interaction with multiple users. In Proc. UIST, (2004), 137-146.

20. Yamashita, N. and Ishida, T. Effects of Machine Translation on Collaborative Work. In Proc. CSCW(2006), 515-524. 